\title{
L'intégration et la mise en œuvre des principes marchands dans le secteur de l'eau en milieu rural et semi-urbain béninois
}

Une analyse des réformes et des pratiques

Héloïse Valette, Fabrice Gangneron et Alain Bonnassieux

\section{OpenEdition}

\section{Journals}

Édition électronique

URL : http://journals.openedition.org/anthropodev/547

DOI : 10.4000/anthropodev.547

ISSN : 2553-1719

Éditeur

APAD - Association pour l'anthropologie du changement social et du développement

\section{Édition imprimée}

Date de publication : 1 mai 2017

Pagination : 113-142

ISBN : 979-10-93476-04-9

ISSN : 2276-2019

Référence électronique

Héloïse Valette, Fabrice Gangneron et Alain Bonnassieux, «L'intégration et la mise en œuvre des principes marchands dans le secteur de l'eau en milieu rural et semi-urbain béninois », Anthropologie \& développement [En ligne], 45 | 2017, mis en ligne le 01 novembre 2017, consulté le 30 avril 2019. URL : http://journals.openedition.org/anthropodev/547 ; DOI : 10.4000/anthropodev.547

La revue Anthropologie \& développement est mise à disposition selon les termes de la Licence Creative Commons Attribution 4.0 International. 


\title{
L'intégration et la mise en œuvre des principes marchands dans le secteur de l'eau en milieu rural et semi-urbain béninois
}

\author{
Une analyse des réformes et des pratiques
}

\author{
Héloïse Valette, Fabrice Gangneron, Alain Bonnassieux
}

Les réformes du secteur de l'eau potable en milieu rural et semiurbain au Bénin ont globalement suivi les recommandations des agences d'aide internationale, en conformité avec le "référentiel marchand" de l'eau (Baron et Maillefert, 2011). Celui-ci est caractérisé par plusieurs principes: (1) la décentralisation des compétences aux communes; (2) la délégation de la gestion du service d'eau à des opérateurs privés; (3) la tarification de l'eau et son paiement systématique au volume; et (4) la participation des acteurs dans la gestion de l'eau. Cet article vise à analyser l'intégration et la mise en cuuvre des principes du référentiel marchand dans les réformes, et à analyser comment ils sont reçus par les acteurs du secteur à différentes échelles territoriales. Nos enquêtes qualitatives menées au niveau national et dans les communes de Djougou et de Kpomassè montrent qu'il ne semble pas y avoir de remise en cause des principes mêmes du référentiel marchand par les acteurs du processus de décision et ceux qui sont chargés de leur mise en ceuvre. Cela ne signifie pas pour autant que ces principes ne suscitent pas de résistances ni de controverses dans leur mise en application.

Drinking water sector reforms in Benin rural and semi-urban areas have broadly met the recommendations of international aid agencies, in compliance with "water market-based referential" (Baron and Maillefert, 2011). This latter has several principles: (1) the decentralization of municipalities' powers; (2) the transfer of water management to private operators; (3) water pricing and charging according to volume of consumption; and (4) the involvement of all stakeholders in water management. Our article seeks to analyze the implementation and integration of these principles of water market-based referential within reforms, as well as examining at different territorial levels the way stakeholders 
assess this process. Our qualitative surveys conducted nationwide and in Djougou and Kpomassè municipalities suggest that stakeholders in decision-making and implementation do not really challenge water market-based referential principles. However, there are controversies and tensions in the way these principles are carried out and implemented.

\section{Introduction}

Depuis le début des années 1990, le secteur de l'eau potable en zones rurales et semi-urbaines ${ }^{1}$ au Bénin est marqué par deux vagues de réformes successives qui concourent à redéfinir le périmètre d'action de l'État. La première s'inscrit dans un processus qualifié de " retrait » ou de " désengagement ${ }^{2}$ de l'État du secteur de l'eau en milieu rural et semiurbain à la suite des programmes d'ajustement structurel (PAS) ${ }^{3}$ au début des années $1990^{4}$. Elle s'inscrit également dans le contexte du bilan de la Décennie internationale de l'eau potable et de l'assainissement, lancée en 1981 à l'initiative de l'ONU, bilan lors duquel la trop grande centralisation des décisions en matière de gestion de l'eau et le manque d'implication des populations dans cette gestion ont été dénoncés (Hounménou, 2006). Avec cette réforme, formalisée par l'adoption de la première stratégie nationale du secteur de l'eau en $1992^{5}$, certaines prérogatives assurées auparavant par l'État central sont déléguées aux communautés locales, suivant une approche dite "par la demande " (évaluation de leurs besoins

${ }^{1}$ Jusqu'à 10000 habitants (Comité de pilotage du Livre bleu Bénin, 2009).

${ }^{2}$ Processus qui par ailleurs peut être considéré comme une phase de « redéploiement de l'État ", où celui-ci fait preuve de résistance et continue de se former à travers la renégociation permanente entre le public et le privé (Hibou, 1999).

3 Le Bénin a signé trois programmes d'ajustement structurel, respectivement dans les périodes 1989-1991, 1992-1994 et 1995-1997.

${ }^{4}$ Les centres urbains, gérés historiquement par une entreprise publique aujourd'hui nommée la Société nationale des eaux du Bénin (SONEB), n'ont pas été concernés par ce processus.

${ }^{5}$ " Stratégie de développement du secteur de l'alimentation en eau et de l'assainissement en zone rurale $"$. 
en matière de points d'eau, participation au financement initial des infrastructures, maintenance des points d'eau).

La seconde vague de réformes se déroule à partir des années 2000, à la suite des lois de décentralisation. La "Stratégie nationale de l'approvisionnement en eau potable en milieu rural du Bénin 2005-2015 » (SNAEP) est définie en 2005 (Direction générale de l'eau, 2005). Les communes deviennent propriétaires des points d'eau, mais la délégation de leur gestion à des opérateurs privés est fortement encouragée par les décideurs. En parallèle, le ministère de l'Eau décide de privilégier de manière quasi systématique les infrastructures d'eau complexes - de type adduction d'eau villageoise - et d'abandonner la construction de nouveaux forages équipés de pompes à motricité humaine (FPMH $)^{6}$.

Du fait de la grande dépendance du pays à l'aide ${ }^{7}$, ces réformes sont empreintes des principes marchands diffusés par les organisations internationales d'aide au développement. Lors de la Conférence internationale de l'eau et de l'environnement de 1992 à Dublin, le principe selon lequel "l'eau, utilisée à de multiples fins, a une valeur économique et devrait donc être reconnue comme bien économique " a été énoncé (ONU, 1992). Ce principe est souvent considéré comme l'illustration la plus criante des orientations marchandes véhiculées par les organisations internationales d'aide au développement dans le secteur de l'eau. En effet, reconnaître la valeur économique de l'eau revient à glisser d'une conception gratuite - ou quasi gratuite - de l'eau à une conception en

${ }^{6}$ Plusieurs types d'ouvrages hydrauliques coexistent dans les territoires ruraux et semiurbains : les puits (publics ou privés) et les forages équipés de pompes à motricité humaine (FPMH) sont considérés comme des " ouvrages simples ». Les FPMH sont des forages équipés de pompes manuelles ou à pédales. Les adductions d'eau villageoise (AEV) sont considérées comme des " ouvrages complexes » car elles nécessitent des forages, des moteurs d'exhaure, des châteaux d'eau et des compteurs de volume. Elles desservent plusieurs bornes-fontaines et des branchements privés.

7 En 2013, deux tiers du budget du secteur de l'eau proviennent des bailleurs de fonds (DG Eau, 2013a). Un grand nombre d'acteurs extérieurs (bailleurs de fonds, ONG, experts internationaux) interviennent dans l'élaboration, l'appui et la mise en œuvre des réformes. Ils n'apportent pas uniquement une aide technique et financière, mais jouent un rôle crucial dans la fabrique des politiques publiques (Lavigne Delville, 2015). 
termes de service d'eau payant. Dans cette optique, un marché peut et doit donc être constitué pour fournir de l'eau aux populations.

Les réformes successives du secteur de l'eau en milieu rural et semiurbain au Bénin semblent ainsi conformes à la montée de ce que Baron et Maillefert (2011) ont conceptualisé sous la terminologie de "référentiel marchand ", que nous discutons dans la première partie de cet article et dont nous retenons quatre principes de mise en œuvre: la décentralisation des compétences aux communes, la délégation de la gestion du service d'eau à des opérateurs privés, la tarification de l'eau et son paiement systématique au volume, ou encore la participation des acteurs dans la gestion de l'eau.

Toutefois, cette conformité a priori ne nous renseigne pas sur la façon dont ces principes ont été traduits dans ces réformes, ni sur les ajustements réalisés par rapport à ces principes, ou encore sur la manière dont les acteurs aux échelles nationales et locales se les approprient, les contournent ou les rejettent. Cet article vise donc à analyser l'intégration et la mise en œuvre des principes du référentiel marchand dans les réformes, ainsi qu'à comprendre comment ils sont reçus par les acteurs du secteur aux différentes échelles territoriales. Nous avons interrogé les acteurs gouvernementaux (au sein des directions ministérielles et déconcentrées), les bailleurs de fonds, les ONG et associations, ainsi que les acteurs du secteur privé (bureaux d'études, experts, entreprises de forage, entrepreneurs). Pour confronter ces réformes aux réalités de terrains, nous avons mené des enquêtes dans deux communes, Djougou et Kpomassè, en raison de leurs situations contrastées sur le plan géographique, économique et du développement des systèmes d'adduction d'eau potable. Nous avons conduit des entretiens semidirectifs auprès des autorités déconcentrées et décentralisées, des ONG locales, des associations d'usagers de l'eau, des acteurs locaux (fontainières, exploitants) et des usagers. Ces données ont permis d'analyser les stratégies des acteurs dans l'acceptation, le rejet ou le contournement des réformes. 
Le " référentiel marchand » comme grille de lecture des réformes et des pratiques

Le recours au concept de référentiel permet de proposer une grille d'analyse des fondements qui guident les activités en matière d'aide internationale. Dans le champ de la science politique, Muller (2010) définit le référentiel comme un " ensemble de prescriptions qui donnent du sens à un programme d'action en définissant des critères de choix et des modes de désignation des objectifs ". En économie institutionnaliste, Baron et Maillefert (2011) ont identifié deux référentiels relatifs à la gestion de l'eau en Afrique francophone, le "référentiel marchand" et le "référentiel hiérarchique ». Ceux-ci sont entendus comme des "valeurs de référence " ou des "idéaux types " qui se traduisent dans les modes d'actions - notamment via les réformes - et dans les pratiques. Le "référentiel hiérarchique " repose sur une régulation fondée sur la hiérarchie, soit sous l'autorité de l'État, soit sous celle de la communauté locale. De leurs points de vue, il prévaut dans les années 1970-1980 et se fonde sur un principe d'accès à l'eau comme un droit, grâce à la possibilité d'un accès minimal gratuit ou subventionné.

Le "référentiel marchand" se décline en deux catégories: le référentiel marchand " pur », guidé par les principes de concurrence pure et parfaite, et le référentiel marchand " amendé ». Ils caractérisent l'eau comme bien à usage marchand et se basent sur des règles d'allocation de l'eau par les prix et le principe d'efficacité. Ce référentiel est dit " amendé » lorsqu'il inclut des mécanismes de correction des défauts du marché.

Dans le secteur de l'eau en milieu rural et semi-urbain, le référentiel marchand amendé se traduit par une contractualisation entre un entrepreneur gestionnaire d'adductions d'eau villageoise (AEV) et une collectivité territoriale et, dans certains cas, des représentants des usagers. Les lois de décentralisation octroyant aux communes l'autorité du secteur ont amorcé ces dynamiques. La commune et les services étatiques 
nationaux, comme la Direction générale de l'eau (DG Eau) ${ }^{8}$, ou départementaux (services de l'Eau), conservent des marges de manœuvre. Ils exercent en particulier des fonctions de coordination et de contrôle. II ne s'agit donc pas d'un retrait de l'État, mais d'une redéfinition de ses fonctions, non plus en tant qu'opérateur mais en tant " qu'organisateur ». Selon ce référentiel, les coûts doivent être pris en charge par les usagers visant ainsi un équilibre de l'exploitation, selon le principe du " sustainable cost recovery" (Camdessus et Winpenny, 2003). II est toutefois " amendé " dans la mesure où les règles purement marchandes ne sont pas appliquées stricto sensu. Par exemple, les coûts de construction des AEV ne sont pas supportés uniquement par les usagers; des politiques de " tranches sociales" sont mises en place - pour lesquelles les premiers mètres cubes consommés aux branchements privés sont à coût réduit - ; ou encore des coûts des extensions d'AEV sont supportés par les communes.

Ce référentiel marchand, dans sa version amendée, semble s'être imposé au niveau international (Baron et Maillefert, 2011 ; Valette et al., 2015). Nous nous intéressons ici aux quatre principaux principes clés de sa mise en œuvre. Le premier principe concerne la tarification du service d'eau et son paiement individualisé et au volume. Derrière ce principe se trouve l'hypothèse selon laquelle payer l'eau permettrait de garantir la pérennité, la qualité et le développement des services (Breuil, 2004), ainsi que de "responsabiliser " les usagers. La tarification est même défendue au nom de l'équité sociale lorsqu'elle inclut des mécanismes redistributifs de type subventions "croisées" ou "indirectes " ${ }^{9}$. Si le principe de tarification du service d'eau n'est jamais remis en cause ou discuté dans la littérature produite par les organisations internationales de l'aide, il l'est dans la sphère académique. Certains auteurs (Vincent, 2003 ; Jaglin, 2005)

\footnotetext{
${ }^{8}$ La DG Eau est l'une des trois directions du ministère de l'Eau en charge notamment de l'eau potable. Le ministère de l'Eau a connu plusieurs dénominations. Aujourd'hui, il est intitulé ministère de l'Énergie, de l'Eau et des Mines.

${ }^{9}$ Le principe de ces subventions repose sur le fait qu'une catégorie d'usagers (les personnes aisées ou grandes consommatrices par exemple) payent leur eau plus cher afin d'en diminuer le prix pour les personnes démunies (cas des tarifications de type progressif) (wikiwater.fr, 2017).
} 
apportent un regard critique sur l'efficacité des mécanismes tarifaires, notamment en ce qui concerne les risques de marginalisation des populations aux faibles pouvoirs contributifs.

Le deuxième principe est lié aux processus de décentralisation. II soustend l'idée que restreindre certaines prérogatives de l'État central permettrait de redonner le pouvoir aux échelons locaux. Les arguments en faveur de la décentralisation sont nombreux, mais toujours supposés : elle serait démocratique, permettrait une plus grande participation locale, améliorerait l'équité au sein des territoires, renforcerait l'efficacité économique et administrative, améliorerait la transparence et la redevabilité des autorités publiques vis-à-vis de leurs populations (Ribot, 2007). Certains auteurs dénoncent aussi le récit dépolitisant construit autour de la décentralisation. Jaglin et Dubresson (1993), par exemple, indiquent que les luttes de pouvoir au niveau national n'ont en rien perdu de leur acuité avec la décentralisation, elles ont simplement été délocalisées. Otayek (2005) souligne que, si les réformes de décentralisation ont été imposées par les agences d'aide internationales, elles ont fait l'objet, pour paraphraser l'auteur, d'un processus d'appropriation, de contournement et d'instrumentalisation par les élites politiques, les réduisant à de "simples problèmes de gestion et de techniques administratives déconnectées des enjeux politiques pourtant inhérents à toute réforme ».

Le troisième principe porte sur la délégation de la gestion de l'eau à un opérateur privé, opérateur supposé plus efficient qu'un opérateur public ou qu'une communauté d'usagers. La gestion déléguée est en effet censée apporter l'efficacité qui serait déficiente dans le modèle de gestion publique ou communautaire. Elle permettrait de déployer de nouvelles compétences, de renforcer l'autonomie locale et de générer de nouvelles ressources financières pour les collectivités territoriales par la levée de taxes sur l'eau. Elle se réalise au travers de la contractualisation entre un opérateur chargé de la gestion et la commune, propriétaire des ouvrages (Baron et Maillefert, 2011). Le bien-fondé supposé du recours à un opérateur privé reste pourtant discuté, y compris au sein des organisations 
internationales comme la Banque mondiale ${ }^{10}$. Dans la littérature, le manque de prise en compte des besoins locaux est souligné. Par exemple, pour Darbon (2007), le modèle de promotion du secteur privé est encouragé face à la défaillance des services publics, bien que les choix politiques et les options technologiques puissent être en déphasage avec les moyens et les besoins des sociétés africaines.

Le quatrième principe concerne la " participation de tous les acteurs du secteur " (ONU, 1992) comme condition d'une " bonne " gouvernance de l'eau. Dans ce cas, ce principe est surtout préconisé au niveau international pour les pays en développement. L'imprécision de cette notion a largement été critiquée dans la littérature académique (Jaglin, 2005 ; Trémolet et Binder, 2010 ; Otayek, 2002). Baron (2007) souligne que la participation est devenue un nouveau principe de développement, mais qu'elle est souvent confinée dans les faits à l'opérationnalisation de règles définies au niveau global, le local étant pris en compte a posteriori. De plus, l'approche participative peut être qualifiée "d'idéalisée " ou de " dépolitisée ", car elle méconnaît les rapports de pouvoirs (Otayek, 2005) et la prégnance de rapports hiérarchiques dans les sociétés ouestafricaines (Baron et Bonnassieux, 2011).

Ces principes et leurs hypothèses d'efficacité du marché, bien que remis en cause par de nombreux travaux, n'en restent pas moins centraux dans les discours de développement des organisations internationales. Dès lors, partir de ces principes nous semble adapté pour analyser comment et par qui le référentiel marchand est intégré dans les réformes et mis en œuvre en milieu rural et semi-urbain béninois. Les jeux d'acteurs hétérogènes (administratifs, privés, etc.) aux intérêts différents sont de même une source de compréhension des enjeux convergents ou divergents autour de l'acceptation, du rejet, du contournement ou de l'appropriation de certains de ces principes.

\footnotetext{
${ }^{10}$ On peut citer par exemple le rapport Marin (2009) de la Banque mondiale, qui porte sur le bilan des partenariats public-privé dans les villes des pays en développement. II indique que le bilan de certaines concessions est mitigé en ce qui concerne l'accès à l'eau.
} 
Traductions et interprétations des principes marchands dans les réformes du secteur

Les principes marchands commencent à être diffusés dans les années 1990 lors de la première vague de réformes. Si les jalons sont posés, ces principes seront opérationnalisés à partir du milieu des années 2000 selon des modalités de mise en œuvre contrastées.

La décentralisation au ccur des réformes : des résistances au niveau national autour de l'arrivée d'un nouvel acteur dans le secteur, les communes

Au Bénin, la décentralisation est formellement inscrite dans la Constitution du 11 décembre $1990^{11}$ même $\mathrm{si}$, avant cette date, de premières expérimentations de décentralisation avaient été engagées dans le pays (Le Meur, 1998). Le processus de décentralisation est officialisé avec la promulgation des trois principales lois de décentralisation en $1999^{12}$ et avec les premières élections communales au début de l'année 2003.

Dans le cadre de la décentralisation, les communes deviennent propriétaires des ouvrages hydrauliques. Elles sont chargées de l'organisation de la distribution de l'eau (loi $n^{\circ}$ 97-029), prérogatives jusqu'alors ministérielles. Le ministère de l'Eau doit les accompagner et les assister dans cette mission.

Cette réforme de décentralisation apparaît dans une certaine mesure en continuité avec la première vague de réformes des années 1990. En effet, la Stratégie nationale de l'eau de 1992, au travers de "l'approche par la demande ", met les communautés rurales au centre du dispositif de gestion de l'eau. Les populations doivent faire des demandes auprès des services de l'État pour obtenir un nouveau point d'eau en contrepartie d'une contribution (souvent symbolique) à l'investissement initial. La

\footnotetext{
${ }^{11}$ Loi n 90-032 du 11 décembre 1990 portant Constitution de la République du Bénin.

12 Lois du 15 janvier 1999 portant Organisation de l'administration territoriale de la République du Bénin (loi $n^{\circ}$ 97-028), Organisation des communes en République du Bénin (loi $n^{\circ}$ 97-029) et Régime financier des communes en République du Bénin (loi n 98-007).
} 
gestion des points d'eau est confiée à des associations d'usagers de l'eau (AUE) pour les adductions d'eau villageoise (AEV) et à des comités de gestion pour les forages équipés de pompes à motricité humaine (FPMH). Les réformes de décentralisation et la deuxième stratégie nationale de l'eau en milieu rural de 2005 maintiennent le principe de participation des communautés instauré par l'approche par la demande pour les requêtes de réalisation et de réhabilitation des points d'eau. En revanche, cet aspect disparaîtra des principes directeurs de la SNAEP actualisée en 2013 au profit des communes qui ont désormais à leur charge les activités de planification des ouvrages "à partir des besoins des localités " (DG Eau, $2013 b)^{13}$.

À partir de la promulgation des lois de décentralisation et des premières élections communales, les organisations communautaires de gestion des points d'eau (AUE, comités de gestion) ont l'obligation de rétrocéder la propriété des ouvrages à la commune, désormais maître d'ouvrage. Les " ouvrages complexes " doivent ensuite faire l'objet d'un contrat entre la mairie et un délégataire. Indiscutablement, cette nouvelle configuration institutionnelle bouleverse les modalités d'action antérieures, plaçant la commune au centre du système de gestion de l'eau.

Dans les faits, au niveau national, le processus de décentralisation a produit des résistances de la part de certains acteurs ministériels. Les résistances portent sur les transferts financiers et techniques des services du ministère de l'Eau vers les communes ${ }^{14}$. Sur le plan financier, un responsable d'une association d'appui aux communes indique que, en 2013, seulement $3 \%$ du budget municipal provenait de l'État central, en raison d'un manque de volonté politique, mais également de contraintes fortes liées aux décaissements au niveau du ministère des Finances. De ce fait, elles restent largement tributaires des financements par projet de la coopération internationale, ce qui altère la continuité de leurs actions.

\footnotetext{
${ }^{13}$ Le renforcement des prérogatives des communes et de leur autonomie dans cette SNAEP de 2013 sera le principal changement de ce texte.

${ }^{14}$ Ce constat est relativement fréquent dans les pays d'Afrique de l'Ouest (voir par exemple Kassibo (2006) pour le Mali ou Dia (2006) pour le Sénégal).
} 
Sur le plan technique et organisationnel, certaines prérogatives censées être du ressort des communes sont conservées par le niveau central ou régional. C'est le cas par exemple de l'organisation des passations de marchés pour la construction d'ouvrages, chapeautée par le ministère des Finances, et ce malgré la dénonciation de nombreuses irrégularités dans la sélection des prestataires. Selon des témoignages d'entrepreneurs, de bailleurs de fonds et d'un élu en 2013, les procédures sous-tendent des enjeux financiers importants. Un grand nombre de bailleurs du programme "Initiative Eau », dont l'objectif était de réaliser 500 AEV d'ici 2015, se sont d'ailleurs retirés pour des problèmes de « mauvaise gestion » lors de l'attribution des marchés. Décentraliser cette compétence signifie donc aussi abandonner certaines activités pouvant être lucratives.

L'administration centrale et ses services départementaux justifient la conservation de certaines prérogatives censées être du ressort des communes car ils estiment que celles-ci ne leur communiquent pas suffisamment d'informations sur la gestion et la distribution de l'eau. Ils dénoncent également un manque de traçabilité des financements transférés et, plus globalement, un manque de compétences des services municipaux. II semble aussi que le personnel communal n'ait pas suffisamment accès à des sources fiables d'information sur les performances des sous-traitants ou sur le suivi de la qualité de l'eau, ce qui l'empêche de mener à bien ses missions (ministère néerlandais des Affaires étrangères, 2011).

On constate enfin un défaut de coordination entre les ministères lié à des activités institutionnelles menées "en silo", au sein d'une même direction, ou entre les différentes directions ministérielles, dans la conduite du transfert de compétences aux communes. Par exemple, selon leurs agents, le ministère de l'Eau et le ministère de la Santé communiquent et coopèrent peu. Le ministère de l'Eau est chargé de définir la politique de l'eau, de veiller à sa mise en œuvre et de suivre la réalisation et le fonctionnement des ouvrages hydrauliques sur tout le territoire. Le ministère de la Santé est chargé de l'assainissement, de I'hygiène et du suivi de la qualité de l'eau consommée. La Direction générale de l'eau (DG Eau) a amorcé avant la Direction nationale de la santé publique le transfert aux communes des activités de sensibilisation 
des communautés. À cause de ce décalage, les actions réalisées aux échelons communaux et locaux manquent de cohérence. Par exemple, en 2013, à Djougou, les structures communales liées à l'eau et à l'hygiène ont chacune un plan d'action communal, respectivement le Plan communal eau et le Plan d'hygiène et d'assainissement, sans coordination prévue de leurs activités de sensibilisation. Ces logiques peuvent être exacerbées par les recherches de financements extérieurs qui s'organisent par directions ministérielles et qui contribuent au cloisonnement sectoriel de l'action publique.

Ainsi, si le principe de décentraliser n'est pas contesté en tant que tel, les résistances du personnel ministériel se cristallisent autour des conditions préalables du transfert de compétences aux communes. Ces résistances ne sont pas toujours explicitées, elles s'illustrent au niveau central par des réflexes corporatistes, comme des rétentions d'information ou la conservation de prérogatives normalement provisoires et de leurs moyens associés. Ces dynamiques témoignent d'une décentralisation inaboutie.

\section{La " professionnalisation du secteur " impulsée au niveau} national via une interprétation sélective de la gestion déléguée au secteur privé

Le référentiel marchand se traduit dans la mise en application du principe de gestion déléguée de l'eau à un opérateur privé à travers la contractualisation. Ce principe est largement soutenu par les acteurs de l'aide, mais selon des conditionnalités et modes d'intervention différents ${ }^{15}$. II s'opérationnalise à partir de 2006 dans le secteur de l'eau au Bénin. II s'agit d'un revirement important par rapport aux années 1990, période durant laquelle les décideurs privilégiaient une gestion déléguée à des communautés d'usagers, considérées alors comme les plus aptes à

${ }^{15}$ La Banque mondiale se positionne sur l'appui au secteur privé et la promotion de technologies innovantes de suivi des points d'eau. La GIZ (Agence allemande pour la coopération internationale), hébergée dans les locaux de la DG Eau, privilégie l'assistance technique dans la gestion déléguée et le "renforcement de capacités 》 des acteurs ministériels et communaux. L'Union européenne finance la réalisation des ouvrages complexes. 
entretenir et à pérenniser le service d'eau. C'est d'ailleurs l'un des aspects de la réforme qui, bien que relativement consensuel à l'échelle nationale, a suscité de nombreuses controverses au niveau local.

Pour légitimer ce principe de gestion déléguée au privé, la DG Eau s'appuie sur des lieux de débats, comme des ateliers nationaux. Lors d'un atelier en $2006^{16}$ rassemblant les cadres de la DG Eau, les représentants de " partenaires techniques et financiers", d'associations d'usagers de l'eau (AUE), du secteur privé, d'ONG ou encore des maires, sont soulignés : l'absence de contrat de maintenance, le " manque de compétences et de sérieux " de certaines structures communautaires, ou encore le " manque de rigueur " dans la gestion financière (DG Eau, 2006; entretiens ministère). Pour autant, les exemples de succès de certaines AUE n'ont pas été notifiés. L'année suivante, lors d'un autre atelier organisé par la DG Eau, les AUE ne seront plus conviées (DG Eau, 2007). Au cours du dernier atelier, l'accent est mis sur la professionnalisation de la gestion des AEV - sous-entendue par le privé - reconnue comme " une meilleure alternative à la gestion communautaire qui a été jugée défaillante » (DG Eau, 2007). Ainsi, les résultats de ces ateliers serviront de justification à l'exclusion progressive des associations d'usagers de l'eau de la gestion et de la vente de l'eau au profit du privé. Bien que les décisions soient débattues collectivement, elles découlent de rapports de force inégaux entre acteurs gouvernementaux et acteurs communautaires.

La gestion des ouvrages "en régie directe ", c'est-à-dire effectuée directement par les communes, est également désavouée par les participants de l'atelier de 2006 et, en conséquence, écartée. Les arguments invoqués à leur encontre concernent :

"[leur] manque de compétences, la lenteur administrative, [...] [ainsi que] les risques de politisation de la gestion, de conflits avec les communautés et d'affectation des fonds tirés de l'exploitation à d'autres fins " (DG Eau, 2006).

\footnotetext{
${ }^{16}$ Cet atelier national était organisé par la DG Eau. Il portait sur « la définition d'une gestion pérenne des AEV ».
} 
Cette argumentation semble plus relever de présupposés idéologiques que de faits avérés. Remarquons que cette mesure dépasse les exigences initiales de la loi, qui stipule que la commune " peut déléguer " la gestion des ouvrages hydrauliques, mais qu'elle n'en a pas l'obligation juridique (loi $n^{\circ}$ 97-029, art. 108). Dans les faits, la régie directe peut exister lorsque les AEV sont insuffisamment rentables. À Barienou, une localité de Djougou où l'AEV est déficitaire à cause des faibles consommations, le fermier a démissionné, c'est donc l'agent communal de l'eau qui s'occupe provisoirement de sa gestion.

Peu de controverses entre les bailleurs de fonds et les agents du ministère ont été observées sur l'ensemble de ce processus de disqualification progressive de la régie directe et de la gestion communautaire au profit d'opérateurs privés. En parallèle, la contractualisation comme outil de légitimation de ces orientations est encouragée. La commune contractualise généralement avec un fermier chargé de la distribution, de l'exploitation et de l'entretien du réseau, tandis qu'elle conserve les prérogatives de renouvellement des infrastructures et des extensions de réseau. Le fermier recrute un exploitant chargé d'entretenir le réseau et de prélever régulièrement les revenus de I'AEV. Une autre option d'affermage est de forme tripartite entre la commune, le fermier et l'ancienne AUE, qui doit se constituer en groupement d'intérêt économique (GIE) depuis 2007 (entretiens ministère). Dans ce cas, le fermier gère la production et le GIE la distribution. La dernière possibilité est une délégation par la commune de la gestion et de la distribution de l'eau à un GIE, celui-ci remplaçant les anciennes AUE. La création de GIE révèle une volonté de faire émerger des " marchés de l'eau » au niveau local. Le GIE est en ce sens conçu par les autorités ministérielles comme un dispositif transitoire pour professionnaliser le secteur. Il dispose de certains avantages tels que la dispense de taxes sur l'eau. Si le GIE parvient à gérer la distribution d'eau, il a vocation à se transformer en entreprise.

Plusieurs arguments sont invoqués au niveau ministériel pour justifier ces orientations en faveur du secteur privé. L'argument principal est la pérennisation des investissements (ministère néerlandais des Affaires étrangères, 2011). Ces orientations sont également appuyées par un discours au niveau national autour de la "modernisation" des 
infrastructures. Ainsi, la construction d'adductions d'eau villageoise (AEV) plutôt que de forages équipés de pompes à motricité humaine (FPMH) est privilégiée systématiquement dans les bourgs de plus de 2000 habitants. Elle découle de la volonté de systématiser la vente de l'eau pour créer des " marchés de l'eau » viables, considérés par les agents ministériels et les bailleurs comme des prérequis à l'amélioration de l'accès à l'eau potable rurale et semi-urbaine (les entretiens avec les cadres du ministère, les organismes de coopération, ainsi que les opérateurs privés sont en cohérence sur ce point). Pourtant, cette orientation est décidée alors même que les compétences des opérateurs privés sont encore limitées et hétérogènes. Aussi, peu de réflexions sont menées sur la rentabilité des infrastructures dans des zones à faible densité de population, sur l'opportunité de continuer à implanter d'autres types d'infrastructures comme les FPMH, qui permettraient de fournir de l'eau potable à moindre coût, ou encore sur les capacités de paiement limitées des populations précaires.

\section{L'institutionnalisation du paiement de l'eau au volume par la préférence aux dispositifs techniques complexes}

Au niveau national, le principe du paiement systématique de l'eau au volume s'est institutionnalisé au fil du temps. II est totalement intégré dans les réformes des années 2000 (DG Eau, 2005 ; 2013b). Il a été rendu possible par la préférence accordée aux dispositifs techniques complexes de type $A E V$ au détriment des autres modes d'accès à l'eau.

Dans les années 1990, les usagers sont sollicités par l'État pour participer financièrement à l'investissement initial des infrastructures FPMH et AEV - et pour assurer leur gestion. Les gestionnaires " communautaires " sont bénévoles. Toutefois, la question du paiement émerge pour assurer la maintenance du système. Le prix de l'eau, relativement faible, varie selon les modalités que choisissent les usagers et la complexité des infrastructures. En ce qui concerne les FPMH, le paiement de l'eau peut être exceptionnel, pour financer les dépannages. II peut être forfaitaire - mensuel ou annuel -, ce qui est fréquemment le cas. Ces ouvrages ne disposant pas de compteurs d'eau, le contrôle des débits et le paiement au volume sont difficiles à mettre en œuvre. Ce dernier peut toutefois s'exercer avec des récipients calibrés (bidons, bassines, 
barriques). Dans ces cas-là, les prix sont plus élevés. En ce qui concerne les $A E V$, la question du paiement prend une autre dimension, puisque son fonctionnement quotidien entraîne des coûts plus importants. En effet, la complexité des infrastructures nécessite des frais de fonctionnement (énergie, maintenance et réparation, frais de personnel, etc.). Le paiement du service d'eau devient donc indispensable et il est rendu possible grâce à des compteurs à chaque borne-fontaine et à la vente en bassines calibrées.

Les débats à l'échelle nationale se focalisent essentiellement sur la question de la tarification de l'eau, souvent au détriment d'autres sujets comme sa qualité, la continuité du service, ou le recours à des sources d'eau alternatives potentiellement contaminées. Les fonctionnaires de la DG Eau et les bailleurs de fonds intervenant dans le secteur sont unanimes sur le principe de paiement pour le service d'eau. Les débats portent plus sur son application concrète au niveau local. Lors de nos enquêtes, certains membres d'ONG internationales influentes dans le processus décisionnel ont indiqué que les réformes en matière de tarification étaient trop brutales compte tenu de la capacité et de la disposition des populations à payer le service. Elles mettent aussi en garde contre les risques d'exclusion permanente ou temporaire des populations vulnérables si les coûts sont trop élevés. Ainsi, le principe de tarification est acté au niveau national, mais nous verrons qu'il suscite des controverses dans sa mise en œuvre en zones rurales et semi-urbaines.

\section{La vision de la participation à l'échelle nationale : des usagers gestionnaires aux usagers clients}

Dans les années 1990, la participation des communautés est invoquée, soit pour les faire contribuer financièrement à l'investissement initial des infrastructures (même si le montant est toujours resté symbolique), soit pour rompre avec une vision du secteur qualifiée de " centralisée " par les ministères et les bailleurs de fonds (IOB, 2011). L'idée de "responsabiliser" les usagers en les impliquant dans le processus décisionnel et la gestion des infrastructures est avancée par ces acteurs.

On constate une évolution du sens de la " participation " au niveau des décideurs à partir des années 2000 et une volonté de mettre en place des lieux d'échanges et de débats participatifs "formalisés ". Émerge par exemple le "Groupe sectoriel Eau » (GS Eau), à l'initiative du 
gouvernement et des bailleurs de fonds. Ce groupe comprend des représentants d'ONG internationales, d'associations de défense des communes, du secteur privé, ou encore du Partenariat national de l'eau du Bénin. Sont aussi présents les différents services gouvernementaux de l'eau et de la santé et les bailleurs de fonds. Ce groupe constitue un lieu d'échange d'informations sur le secteur, mais il n'a pas officiellement de pouvoir de décision. Officieusement, il permet d'infléchir certaines orientations ou de mettre à l'ordre du jour des préoccupations des acteurs présents.

En pratique, la participation de tous les acteurs du secteur est relative. Les représentants d'associations villageoises de points d'eau, de chefferies ou encore d'ONG locales sont très peu présents dans ce groupe alors qu'ils sont influents au niveau local (Le Meur, 1998). Du fait de la concentration des acteurs de la participation dans la capitale économique ${ }^{17}$, cette participation s'exerce essentiellement à Cotonou et limite les contributions d'acteurs locaux. De plus, les ONG internationales et les associations locales travaillent en étroite collaboration avec les autorités ministérielles et les bailleurs de fonds, ces derniers finançant une partie importante de leurs activités. Ces interrelations peuvent minimiser leurs éventuelles réserves sur l'orientation marchande des réformes et leur emprise sur le choix des débats en amont. Elles parviennent tout de même à faire remonter certaines préoccupations locales, relatives par exemple aux coûts de l'eau aux AEV, à la discontinuité du service ou encore aux difficultés que connaissent les communes dans la gestion du secteur.

Dans le cadre du processus de "professionnalisation du secteur", la DG Eau a également impulsé, avec l'aide de la GIZ, la mise en place d'associations de consommateurs d'eau potable (ACEP) au niveau communal. Cependant, la structure et les mandats, qui ont été pensés et formalisés au niveau ministériel, font des ACEP des instances participatives imposées ex nihilo au niveau local. Ces dynamiques révèlent aussi l'évolution d'une vision des usagers comme gestionnaires à une vision des usagers comme clients.

${ }^{17}$ La plupart des ONG et associations représentées dans ce groupe ont leur siège à Cotonou. 
À noter que les opérateurs privés se sont organisés et portent leurs requêtes dans les instances décisionnelles et participatives. Ils se structurent grâce à l'Association des fermiers du secteur de l'eau au Bénin (AFEB). Celle-ci parvient à faire entendre la voix d'un secteur privé, sur des sujets comme la subvention à l'implantation de branchements privés pour rentabiliser les installations. Grâce à cette représentation, les fermiers bénéficient d'une écoute et d'un pouvoir d'action relativement importants.

Les réformes du secteur de l'eau définies et mises en œuvre au Bénin sont ainsi en cohérence avec les différents principes du référentiel marchand. Leur mise en application, bien que balbutiante, débute en partie dans les années 1990 et se concrétise dans les années 2000-2010. Cette mise en œuvre n'est pas mécanique et est sujette à des interprétations. L'ensemble de ces dynamiques peut s'expliquer par un soutien extérieur important. Néanmoins, cette homogénéité apparente sous-tend des controverses et disputes entre les acteurs aux différentes échelles. Celles-ci sont toutefois contenues pour ne pas dénoncer un système d'aide dont ils dépendent. Elle sous-tend également des interprétations variées de ces principes. Parfois, ceux-ci sont interprétés de manière extensive, comme c'est le cas pour la délégation de la gestion du service à des opérateurs privés. Parfois, ces principes connaissent une évolution de leurs sens, comme c'est le cas avec l'interprétation de la notion de participation au fil du temps.

Djougou et Kpomassè, une traduction marchande hétérogène, mais effective

La traduction des principes marchands diffère selon les contextes et les acteurs locaux en présence. Cette partie vise à confronter les réformes aux réalités de terrains dans deux communes aux situations contrastées, Djougou, au centre-nord du pays, et Kpomassè, au sud-ouest. Djougou a un taux de couverture beaucoup plus faible que Kpomassè. En effet, les chiffres officiels de la DG Eau de 2014 annoncent un taux de couverture de $58,1 \%$ en zone rurale pour Djougou contre $99,1 \%$ pour Kpomassè ; ces chiffres ne tiennent néanmoins pas compte du taux de pannes. 
À Djougou, la couverture rurale des AEV reste modeste, mais en progression. Dans cette commune, l'agriculture est l'activité dominante en dehors du chef-lieu. Kpomassè, une commune proche de Cotonou, bénéficie d'une expérience plus aboutie en matière d'affermage. Les consommations d'eau aux AEV y sont plus importantes. En effet, la commune jouit d'activités économiques diversifiées, elle accueille de petites et moyennes entreprises et bénéficie d'un nombre nettement plus élevé de branchements privés.

Rôle des communes, réalités de la gestion déléguée et repositionnement des acteurs lors des réformes

Le référentiel marchand s'opérationnalise au travers des réformes de décentralisation et de la délégation de la gestion de l'eau à des opérateurs privés. Plusieurs tendances issues des enquêtes de terrain peuvent être dégagées.

Des dynamiques communales entachées par des difficultés opérationnelles

Les services techniques des communes sont en sous-effectifs et leurs dotations en deçà de leurs besoins. Toutefois, les personnels affectés aux deux communes, en particulier les chefs de services techniques (CST) ont une bonne connaissance du fonctionnement du secteur et prennent des initiatives pour tenter de remédier aux difficultés auxquelles ils sont confrontés. Outre les CST, qui sont fonctionnaires, une partie du personnel municipal est tributaire de financements d'organismes de développement, par "projet ». Ce mode de fonctionnement à durée déterminée génère des incertitudes sur les renouvellements des postes. Ces personnels doivent aussi faire face à des initiatives qui court-circuitent leurs services. Des ONG implantent des ouvrages sans accord préalable de la commune, à Djougou comme à Kpomassè. Des particuliers vendent l'eau de leur forage privé meilleur marché que la tarification officielle (Kpomassè). Face à ces initiatives, les communes sont relativement démunies et ont peu de pouvoir de sanction. 


\section{Un processus conflictuel de rétrocession des AEV en voie d'apaisement}

Les communes ont souvent été en conflit avec les notables locaux et les usagers lors de la réforme du secteur. En effet, les responsables des anciennes AUE de Djougou ont ressenti le processus de décentralisation comme une confiscation des ouvrages et des recettes associées, au profit de fermiers extérieurs aux villages (Bonnassieux et Gangneron, 2011). Or, sous gestion communautaire, les recettes de l'eau étaient fréquemment mobilisées pour participer à des actions collectives locales, comme la construction de salles de classe. Les recoupements auxquels nous avons procédés montrent qu'une partie des recettes était probablement captée par les gestionnaires.

Ces conflits semblent s'atténuer aujourd'hui et des arrangements locaux s'opèrent. Certains notables ont adapté leurs stratégies : autrefois membres d'AUE, certains se sont insérés dans des associations de consommateurs (ACEP), tandis que d'autres sont devenus exploitants comme dans la localité de Kolokondé -, voire fermiers.

À Kpomassè, ce processus de rétrocession des ouvrages a été moins conflictuel. Par exemple, une ancienne AUE s'est constituée en groupement d'intérêt économique (GIE) et a spontanément fait appel à un retraité de la Société des eaux du Bénin pour diriger et gérer l'AEV. Cet ingénieur de formation est aujourd'hui le président de l'Association des fermiers de l'eau du Bénin (AFEB). Après une période de transition, le GIE est devenu une entreprise et a conservé la concession. Plusieurs autres exploitants (à Tokpa-Domé et Kpomassè centre) sont d'anciens membres d'AUE reconvertis. Ces exemples sont souvent présentés comme la réussite des processus de mise en affermage, bien qu'ils soient à notre connaissance peu répandus. Notons que selon certains acteurs (exploitant, ancienne présidente d'AEV), les passages AUE/GIE/entreprise privée n'auraient pas nécessairement constitué une amélioration du service d'eau.

À Kpomassè comme à Djougou, la mise en affermage des AEV a suscité des réticences. Des exploitants ont vu pour certains leurs marges de manœuvre et leurs revenus baisser lorsqu'ils sont passés au statut d'employés des fermiers. Quand les associations d'usagers de l'eau avaient 
la gestion des $A E V$, ils occupaient un rôle de premier plan, comme à Kolokondé et à Dékanmey (Bonnassieux et Gangneron, 2011 ; entretiens avec les associations d'usagers et les exploitants).

\section{La délégation de la gestion des AEV en contexte}

La délégation de la distribution de l'eau à des opérateurs privés est effective, mais son fonctionnement n'est que partiellement conforme au modèle de gestion déléguée prévu par le niveau national, et très inégal selon les contextes. Les compétences des fermiers sont extrêmement hétérogènes. L'un d'entre eux travaillait auparavant dans un bureau d'études sur l'eau et l'assainissement et a décidé de diversifier ses activités (Djougou). Un autre est un ancien cadre hydraulique (Kpomassè). Certains découvrent totalement ce métier et sont issus d'autres secteurs, comme le bâtiment. Ils peuvent être originaires de la commune, ou résider à Cotonou. Selon leur profil, la mise en affermage prend des formes plus ou moins pacifiées.

À Djougou, la commune reproche aux fermiers leur manque d'efficience, de communication et leur absentéisme chronique. Certaines $A E V$, non rentables en l'état, ont même été abandonnées. Les consommations d'eau $\mathrm{y}$ restent faibles et saisonnières du fait des modestes capacités à payer des ménages et de la présence de sources alternatives moins chères, voire gratuites (FPMH, puits, eaux de surface ou de ruissellement). Certains fermiers dénoncent aussi des tentatives de captation des recettes de l'eau par la commune, un cadre juridique trop fragile et des contrats de durées parfois extrêmement courtes (de seulement deux ans) les dissuadant d'investir dans l'entretien du réseau.

Des tensions apparaissent aussi entre certains fermiers et leurs exploitants. Ces derniers sont parfois livrés à eux-mêmes - sans pièces de rechange ni fonds propres - pour maintenir le service. Ils sont irrégulièrement et peu rémunérés. Malgré leurs conditions précaires, certains parviennent à maîtriser les incertitudes d'une fonction dont le formalisme contractuel reste abstrait, à assurer une continuité du service dans les villages de la commune. Par exemple, pour acheter des pièces détachées, ils utilisent directement l'argent collecté aux bornes-fontaines, sollicitent des dons auprès des notables locaux, ou recrutent de jeunes villageois pour procéder aux réparations qu'ils paient sur les recettes. 
À Kpomassè, la délégation au privé est l'une des plus avancées du pays. Les fermiers sont plus présents qu'à Djougou. Les exploitants y suivent des procédures plus formalisées, les cadrages de leurs fonctions y sont plus rigoureux (journées des tournées, procédures de suivi des dispositifs, gestion des pannes, etc.). Le marché de l'eau est viable en raison du caractère semi-urbain des localités et d'un volume élevé des consommations. Les branchements individuels sont plus nombreux. Lorsque le marché n'est pas viable, c'est en raison d'une mauvaise implantation des bornes-fontaines, éloignées des habitations, de la vétusté du réseau, de compétences techniques limitées, ou encore du manque de pièces détachées pour effectuer les réparations en cas de pannes.

Dans les deux cas, les désaccords et disputes sont fréquents, quels que soient les acteurs : les communes peinent à recouvrer les taxes dues par les fermiers, tandis que les fermiers dénoncent leur niveau trop élevé ou des AEV livrées en médiocre état. Les exploitants accusent les fermiers de les payer insuffisamment. Ces disputes ne vont cependant jamais jusqu'à la rupture. En effet, chacun tente de tirer profit de ce marché émergent en améliorant ses positions. Les communes montrent une flexibilité dans la levée des taxes : tous les fermiers, à Djougou comme à Kpomassè, sont en retard de paiement, mais les communes proposent toujours des arrangements (échelonnement des remboursements, allègement des paiements). Les employés acceptent de travailler dans des conditions précaires et dégradées.

Dans les deux communes, les anciens responsables d'AUE, généralement des notables locaux, ont souvent su adapter leurs rôles au fil du temps, en s'insérant dans les nouvelles organisations comme les GIE. Certains fermiers ont donc bénéficié de compétences communautaires qui les avaient précédées. Ainsi, cette gestion délégataire, à première vue en rupture avec les modalités d'action communautaires antérieures, révèle une certaine continuité avec la gestion déléguée au privé.

\section{Une tarification de l'eau moins acceptée à Djougou qu'à Kpomassè}

Le montant du prix de l'eau, ainsi que son augmentation, ont suscité des controverses au niveau local. 
Le montant des prix aux AEV est bien plus élevé qu'aux forages équipés de pompes à motricité humaine. Selon nos enquêtes, ils sont établis aux FPMH dans une fourchette entre 200 et 500 FCFA par $\mathrm{m}^{3}$ à Kpomassè et entre 150 et 300 FCFA par $\mathrm{m}^{3}$ à Djougou. Les forfaits annuels ou mensuels existent toujours pour ces modes d'accès: à Djougou, un forfait de 1000 FCFA par an est par exemple institué dans une localité. À Partago, le paiement a été établi entre 200 et 500 FCFA par mois, selon les moyens des ménages.

Actuellement, le prix de l'eau aux AEV tend à s'uniformiser sur tout le territoire, mais il reste plus faible à Djougou qu'à Kpomassè. Officiellement, le prix de l'eau est de 500 FCFA par $\mathrm{m}^{3}$ pour les bornesfontaines des AEV et de 580 FCFA si les ménages sont raccordés à domicile sur l'AEV (branchements privés). À Kpomassè, ce prix est de 575 FCFA par $\mathrm{m}^{3}$ pour les bornes-fontaines et les branchements privés. Dans les faits, les usagers paient l'eau au volume à un tarif bien plus élevé en raison de la multiplication des intermédiaires et des frais de gestion. En effet, certaines fontainières accroissent les tarifs de l'eau pour assurer une rémunération un peu plus que symbolique en jouant sur les volumes délivrés. De fait, le prix de l'eau est souvent plus proche de 800 FCFA par $\mathrm{m}^{3}$ que du prix officiellement discuté entre communes et fermiers.

La mise en affermage des AEV et l'encadrement de leur gestion par les communes ont entraîné une augmentation du prix de l'eau. Les intermédiaires dans la chaîne de distribution se sont multipliés (commune, fermier, exploitant, fontainières). Les fermiers entendent dégager des profits, les communes lèvent sur l'eau des taxes auparavant inexistantes. Nos enquêtes à Kolokondé (Djougou) et Dékamney (Kpomassè) montrent que les prix de l'eau aux AEV étaient plus bas avant leur mise en affermage, lorsqu'elles étaient gérées par des associations d'usagers de l'eau et un exploitant au nom de la communauté locale.

Le paiement aux $A E V$ est moins débattu à Kpomassè qu'à Djougou, où le pouvoir d'achat est en moyenne plus faible. C'est d'ailleurs à Djougou que l'augmentation du prix de l'eau aux AEV fait le plus débat. Il génère des disputes au sein même des ménages concernant la répartition de ces nouvelles dépenses. 
Les différences de prix par rapport à ceux pratiqués à Djougou ville administrée par la Société nationale des eaux responsable des zones urbaines, la SONEB, - sont également perçues par les habitants comme une injustice. En effet, alors que le $\mathrm{m}^{3}$ d'eau délivré par les AEV coûte officiellement entre 500 et 580 FCFA, la SONEB fait payer :

- la première tranche (de 0 à $\left.5 \mathrm{~m}^{3}\right) 198$ FCFA par $\mathrm{m}^{3}$,

- la deuxième tranche $\left(\right.$ de 6 à $\left.50 \mathrm{~m}^{3}\right) 453$ FCFA par $\mathrm{m}^{3}$,

- la troisième tranche (au-delà de $50 \mathrm{~m}^{3}$ ) 658 FCFA par $\mathrm{m}^{3}$; elle seule est à un prix plus élevé.

La décentralisation du service d'eau pose ainsi des problèmes de justice spatiale entre zones urbaines et rurales.

\section{Une participation à double vitesse?}

Au niveau local, la participation des usagers à la gouvernance de l'eau se cantonne essentiellement à sa dimension financière: participer, c'est payer l'eau. En effet, les associations d'usagers de l'eau et, plus généralement, les populations semblent peu informées de la stratégie sectorielle et des processus de négociation effectués au niveau central (ministère néerlandais des Affaires étrangères, 2011 ; entretiens usagers). Lors de nos enquêtes de terrain, les ACEP, censées être mises en place au niveau des communes pour relayer les demandes des usagers, n'étaient que rarement invitées à s'exprimer dans l'arène municipale.

À Kpomassè, le sentiment d'exclusion est moins fort qu'à Djougou grâce à la présence d'acteurs " translocaux ", qui font remonter au niveau national les préoccupations locales. C'est le cas du président de l'AFEB, qui, grâce à ses activités d'affermage et à son capital politique et social important, contribue, d'une part, à l'acceptation et l'opérationnalisation des principes marchands au niveau local et, d'autre part, à la remontée des difficultés au niveau communal et au niveau national.

Nombre d'imperfections et de tâtonnements sont ainsi constatés dans la mise en œuvre du référentiel marchand dans ces deux communes. La réorganisation institutionnelle a multiplié les acteurs de l'eau au niveau local. Elle a abouti à de multiples arrangements avec un maintien, voire une augmentation de la diversité des configurations institutionnelles. Les 
incertitudes quant à l'émergence de marchés de l'eau ruraux et semiurbains sont nombreuses. Le système d'affermage est très peu rémunérateur pour les acteurs "du bas » (exploitants et fontainières), voire pour les fermiers eux-mêmes. La délégation de la gestion de l'eau à des privés n'a pas forcément garanti l'efficacité ni la durabilité du service. En revanche, elle a généré une augmentation du prix de l'eau. Toutefois, les orientations prises dans les réformes sont, à l'heure actuelle, globalement acceptées dans les deux cas.

\section{Conclusion}

Malgré les incertitudes sur leur pertinence et leur efficacité, nos enquêtes n'ont pas fait apparaître de remise en cause des principes marchands, ni par les acteurs nationaux qui se chargent de leur traduction dans les réformes, ni par ceux chargés de leur mise en œuvre.

Cela ne signifie pas pour autant que ces principes ne suscitent pas de résistances ni de conflits dans leur mise en application. II en existe à tous les niveaux, autour de la répartition des rôles et des ressources entre les différents acteurs au sein de cette nouvelle configuration institutionnelle et dans un contexte d'aide internationale omniprésente. Le processus décisionnel reste très centralisé. Le ministère de l'Eau élabore un cadre légal conforme aux principes du référentiel marchand, mais conserve la mainmise sur des activités clés. Les mairies se saisissent de leurs prérogatives, mais peinent à percevoir les taxes pour assumer certaines fonctions (animation, extension des équipements) et à s'autonomiser par rapport aux aides techniques et financières internationales. Les fermiers ont souvent des difficultés à rémunérer leurs employés à cause de la rentabilité insuffisante et inégale des adductions d'eau villageoise (AEV). Des résistances de la part d'anciens gestionnaires et notables locaux voient le jour suite aux transferts de la compétence "eau " aux autorités communales. En effet, ceux-ci ne peuvent désormais plus décider de l'affectation des recettes issues de la vente de l'eau. Certains acteurs locaux comme les exploitants qui, à l'ère de la gestion communautaire, occupaient un rôle de premier plan dans le fonctionnement de l'AEV, voient leurs positions sociales souffrir et leurs rétributions baisser à mesure qu'ils deviennent de simples exécutants. Les fontainières estiment 
quant à elles que les tarifs fixés pour les différents récipients calibrés utilisés par les usagers sont trop bas pour que leurs lourdes astreintes quotidiennes au niveau des bornes-fontaines soient rétribuées, même de façon très modeste (Bonnassieux et Gangneron, à paraître). Enfin, les résistances proviennent aussi des usagers, qui doivent faire face à une augmentation conséquente du prix de l'eau, résultant de la préférence donnée aux dispositifs techniques complexes et à la multiplication des intermédiaires lors de la mise en affermage. Ils déploient alors des stratégies afin de réduire les coûts en diversifiant leurs modes d'accès à l'eau (FPMH, puits, eaux de surfaces ou de pluie).

On constate aussi une certaine continuité dans la mise en œuvre des principes marchands par rapport aux modes d'actions communautaires antérieurs. La majorité des acteurs participant à l'élaboration des politiques et à leur mise en œuvre (services de l'hydraulique, ONG internationales et nationales) sont financés en grande partie par des agences d'aide internationale. Le déploiement des AEV concerne désormais l'ensemble des territoires et la tendance commune est à l'émergence de marchés de l'eau. L'acceptation des réformes est aussi facilitée avec le temps, en atteste par exemple la légitimité croissante des communes dans le secteur. Enfin, de nombreux acteurs, en particulier les anciens responsables des associations d'usagers communautaires, ont su modifier leurs modes d'intervention dans cette nouvelle configuration institutionnelle pour ne pas être exclus du processus.

L'ensemble des acteurs a ainsi cherché à se repositionner par rapport à ces nouvelles normes, les uns pour en tirer parti, les autres pour tenter d'en minimiser les conséquences négatives, produisant des configurations de terrain hétérogènes, une qualité de service très inégale. Les principes du référentiel marchand peuvent ainsi sembler être appliqués à l'échelle locale, sans pour autant que la qualité du service n'en soit nettement améliorée, et au prix d'un accroissement du tarif de l'eau pour les populations. 


\section{Bibliographie}

BARON C., 2007, "Société civile et nouvelles formes de partenariat pour l'accès à l'eau dans les pays en développement ", Revue internationale et stratégique, 2(66) : 79-92.

BARON C. et BONNASSIEUX A., 2011, "Les enjeux de l'accès à l'eau en Afrique de l'Ouest: diversité des modes de gouvernance et conflits d'usages ", Mondes en développement, 4(156) : 17-32.

BARON C. et MAILLEFERT M., 2011, "Une lecture institutionnelle de la gouvernance de l'eau potable: des terrains d'Afrique de l'Ouest francophone aux faits stylisés ", Régions \& Cohésion, 1(3) : 7-33.

BONNASSIEUX A. et GANGNERON F., 2011, « Des miniréseaux d'eau potable : entre enjeux politiques et arrangements locaux. Le cas de la commune de Djougou au Bénin ", Mondes en développement, 3(155) : 77-92.

BONNASSIEUX A. et GANGNERON F., à paraître, " Les fontainières des adductions d'eau potable au Burkina Faso et au Bénin, actrices d'une discrète transformation d'une fonction subalterne ", in GUETAT H., TEXIER P., Titre non défini, éd. Quæ.

BREUIL L., 2004, « Renouveler le partenariat public-privé pour les services d'eau dans les pays en développement. Comment conjuguer les dimensions contractuelles, institutionnelles et participatives de la gouvernance ? ", Thèse de doctorat en sciences de gestion, ENGREF, AgroParisTech, Paris, $323 \mathrm{p}$.

CAMDESSUS M. et WINPENNY J., 2003, "Financer l'eau pour tous ", Rapport du Panel mondial sur le financement des infrastructures de l'eau, $62 \mathrm{p}$.

COMITE DE PILOTAGE DU LIVRE BLEU (CPLB) BENIN, 2009, Le livre bleu.

DARBON D., 2007, "Réformer un inexistant désiré ou supprimer un inopportun incontournable? Le service public confronté à l'État et aux sociétés projetées en Afrique ", Téléscope, hiver 2007-2008, 17 p.

DIA A.H., 2006, «Décentralisation et développement local : le cas de la vallée du fleuve Sénégal ", Thèse de Doctorat en sociologie, Université Toulouse 2, $572 \mathrm{p}$.

DIOP M. et HAMATH Dia A., 2011, "Réformes des services d'eau en milieu rural africain : enjeux et limites du montage institutionnel de gestion. Une étude de cas au Sénégal », Mondes en développement, 155(3) : 37-58. 
DIRECTION GENERALE DE L'EAU (DG EAU), 2005, "Stratégie nationale de l'approvisionnement en eau potable en milieu rural du Bénin 20052015 ", ministère des Mines, de l'Énergie et de l'Hydraulique, Cotonou, $21 \mathrm{p}$.

DIRECTION GENERALE DE L'EAU (DG EAU), 2006, « Atelier national sur la définition d'une gestion pérenne des AEV », Rapport de synthèse du MMEE, Cotonou, $11 \mathrm{p}$.

DIRECTION GENERALE DE L'EAU (DG EAU), 2007, «Atelier national sur les modalités de mise en œuvre de la gestion professionnelle des AEV et sur les participations financières initiales », Rapport de synthèse du MMEE, Cotonou, $25 \mathrm{p}$.

DIRECTION GENERALE DE L'EAU (DG EAU), 2013a, « Rapport d'exécution du BPO 2012 de l'approvisionnement en eau potable en milieu rural et de la gestion des ressources en eau ", ministère de l'Énergie, des Recherches pétrolières et Minières, de l'Eau et du Développement des Énergies Renouvelables, Cotonou, $126 \mathrm{p}$.

DIRECTION GENERALE DE L'EAU (DG EAU), 2013b, "Stratégie nationale de l'approvisionnement en eau potable en milieu rural du Bénin 20052015 ", ministère de l'Énergie, des Recherches pétrolières et Minières, de l'Eau et du Développement des Énergies Renouvelables, Cotonou, $41 \mathrm{p}$.

HIBOU B., 1999, La privatisation des États, Éditions Khartala, Coll. Recherches internationales, $400 \mathrm{p}$.

HOUNMENOU B. G., 2006, « Gouvernance de l'eau potable et dynamiques locales en zone rurale au Bénin ", Développement durable et territoires : $19 \mathrm{p}$.

JAGLIN S., 2005, « La participation au service du néo-libéralisme ? Les usagers dans les services d'eau en Afrique subsaharienne ", in BACQUE M-H., REY H., SYNTOMER Y., Gestion de proximité et démocratie participative: une perspective comparative, Éd. La Découverte, Coll. Recherches, Paris : 271291.

JAGLIN S., DUBRESSON A. (dir.), 1993, Pouvoirs et cités d'Afrique noire. Décentralisations en questions, Éditions Karthala, Paris, 312 p.

KASSIBO B., 2006, «Mali : une décentralisation à double vitesse ? : Ka mara la segi so ou le lent et délicat retour du pouvoir à la maison », in FAY C., KONE Y.F., QUIMINAL C. (éds), Décentralisation et pouvoirs en Afrique: en contrepoint, modèles territoriaux français, Paris, Bamako, IRD, ISH : 6795. 
LAVIGNE DELVILLE P., 2015, «Les processus de changement dans les politiques publiques ", in VALETTE H., BARON C., ENTEN F., LAVIGNE DELVILLE P., TSITSIKALIS A., Une action publique éclatée? Production et institutionnalisation de l'action publique dans les secteurs de l'eau potable et du foncier (APPI) (Burkina Faso, Niger, Bénin), Éd. Gret, Nogent-sur-Marne : 72-78.

LE MEUR P.Y., 1998, " Décentralisation par le Bas et Participation clientéliste au Bénin ", Le bulletin de l'APAD, $15: 16 \mathrm{p}$.

MARIN P., 2009, "Partenariats public-privé pour les services d'eau urbains, Bilan des expériences dans les pays en développement ", Rapport pour la Banque mondiale, Washington, $196 \mathrm{p}$.

MINISTERE NEERLANDAIS DES AFFAIRES ETRANGERES, 2011, "Évaluation d'impact des programmes d'approvisionnement en eau potable et d'assainissement au Bénin. Le risque d'effets éphémères ", Rapport, La Haye, $216 \mathrm{p}$.

MULLER P., 2010, "Référentiel ", in L. BOUSSAGUET, S. JACQUOT, RAVINET P., Dictionnaire des politiques publiques, Édition Sciences Po, Coll. Références, $3^{\mathrm{e}}$ éd., Paris : 555-561.

ONU, 1992, Déclaration de Dublin sur l'eau et le développement durable, Dublin.

OTAYEK R., 2002, "Démocratie et société civile. Une vue du sud. Avant-propos ", Revue internationale de politique comparée, 2(9) : 167-170.

OTAYEK R., 2005, « La décentralisation comme mode de redistribution autoritaire ? Quelques réflexions à partir de situations africaines ", Communication au VIII ${ }^{\circ}$ Congrès de I'Association française de sciences politiques à Lyon, 1416 sept. 2005, 13 p.

RIBOT J., 2007, « Dans l'attente de la démocratie : la politique des choix dans la décentralisation de la gestion des ressources naturelles », World Ressource Institute, Washington, 201 p.

TRÉMOLET S., BINDER D., 2010, La régulation des services d'eau et d'assainissement dans les PED. Revue de la littérature, éclairages et pistes de recherche, Coll. À Savoir, AFD, Paris, $111 \mathrm{p}$.

VALETTE H., BARON C., ENTEN F., LAVIGNE DELVILLE P., TSITSIKALIS A., 2015, Une action publique éclatée ? Production et institutionnalisation de l'action publique dans les secteurs de l'eau potable et du foncier (APPI) (Burkina Faso, Niger, Bénin), Éd. Gret, Nogent-sur-Marne, 141 p. 
VINCENT I., 2003, «Le prix de l'eau pour les pauvres: comment concilier droit d'accès et paiement d'un service ? ", Afrique contemporaine, 1(205): 119-134.

\section{Niviv}

Héloïse Valette est doctorante

Sciences Po Toulouse, université Toulouse 1 Capitole, LEREPS

E-mail : heloise.valette@ut-capitole.fr

Fabrice Gangneron est socio-géographe

université Paul Sabatier (OMP), IRD, CNRS, GET

E-mail : fabrice.gangneron@Imtg.obs-mip.fr

Alain Bonnassieux est chercheur au LISST, Dynamiques Rurales

université Toulouse 2 Jean Jaurès, LISST

E-mail : alain.bonnassieux@univ-tlse2.fr 